\title{
Formation par projet et opportunité d'accès à distance à des ressources pédagogiques
}

\author{
Florin HUTU, Bruno ALLARD, Fabrice JUMEL, Mathieu MARANZANA, Kevin MAR- \\ QUET, Lionel MOREL, Luong-Viet PHUNG, Tanguy RISSET, Dominique TOURNIER, Guil- \\ laume SALAGNAC, Jacques VERDIER \\ florin-doru.hutu@insa-lyon.fr \\ Adresse : INSA Lyon, 2 avenue Albert Einstein, F-69621 Villeurbanne Cedex
}

\begin{abstract}
RESUME : Lorsque l'apprentissage par projet couvre plusieurs thématiques, il apparaît que les étudiants sont d'autant plus sensibles à la qualité des ressources mises à disposition et à leur disponibilité. L'évaluation des étudiants est contrastée entre leur plébiscite du contenu de la formation et leur critique des ressources. L'accès à distance à de telles ressources répond à une partie de la problématique. La communication décrit une première expérience de formation par projet qui débouche sur une mise à disposition à distance d'un lot de ressources, assistée d'une pédagogie adaptée. Fort de cette expérience, une seconde proposition est présentée dans un contexte différent mais où des contraintes similaires persistent sur les ressources. La discussion fera l'objet d'un poster avec démonstration sur machine (PR). Il est évident que les propositions mises en cuvre sont maintenant connues, mais peu usitées en fait, et elles sont applicables à d'autres contextes. Si l'opportunité d'accessibilité à distance de ressources est une réponse à une partie importante des faiblesses des retours d'expérience décrits ici, il n'est pas envisagé de formation à distance, problématique très différente.
\end{abstract}

Mots clés : retour d'expérience, accès à distance de ressources

\section{INTRODUCTION}

En 2005, l'INSA de Lyon a ouvert la possibilité de formations optionnelles transversales (OT). La transversalité s'entend à l'intérieur d'un corpus scientifique ou même sur plusieurs corpus scientifiques et techniques. Les systèmes embarqués temps-réels, non enseignés comme tels dans l'établissement, sont apparus comme représentatifs d'une telle transversalité. Une formation de 96h a été construite pour permettre un échange entre des étudiants du département Informatique - peu sensibilisés aux aspects matériel-, du département Génie Electrique - peu sensibilisés aux méthodologies de conception logicielle- et du département Télécommunications et Usages - déjà sensibilisés aux systèmes embarqués par l'aspect communication. Il n'est pas étonnant que peu d'étudiants du département TC soient attirés par cette formation transversale alors que le succès ne se dément pas du côté des deux autres départements (IF et GE).

La formation est intitulée OT SETRE «systèmes embarqués temps-réels ». Elle vise à mettre les étudiants en situation, à savoir de conduire un développement de projet, en usant de méthodologies et pour démontrer une réussite par rapport à un cahier des charges. Les étudiants doivent appréhender un matériel volontairement particularisé (aucun étudiant ne peut avoir de connaissances antérieures complètement utilisables). Les $2 / 3$ des $96 \mathrm{~h}$ sont consacrés au projet, appelé "fil rouge », car différents cours introduisent des notions nécessaires ou complémentaires des cursus originaux, et renvoient directement au projet. La section 2 décrit le contenu des cours et le cahier des charges du projet 2011-2012.

Les ressources matérielles offertes aux étudiants ont évoluées déjà 2 fois suite à l'appréciation des étudiants. Autant l'OT SETRE bénéficie d'un accueil et d'une évaluation extrêmement positive de la part des étudiants, sur son contenu, sa forme et son rythme - bien que les étudiants aient un travail très important à fournir avec un encadrement assez strict -; autant leur critique ne diminue pas depuis 6 ans à propos de la qualité du matériel et de sa disponibilité. Ceci a conduit à envisager 
la création de ressources accessibles à distance. La section 3 décrit ce projet, ses difficultés techniques et financières ainsi que l'impact sur la pédagogie.

Les écueils essuyés par le projet de création de ressources accessibles à distance, ont été intégrés dans la mise en œuvre des ressources d'une autre formation par projet, au sein du département de Génie Electrique. Cette ressource couvre un exemple de transversalité au sein d'un même corpus technique. La section 4 décrit cette ressource et sa prochaine duplication pour la rendre accessible à distance. Il sera conclu que ces ressources, bien que motivées par des objectifs différents, se complètent et incitent à poursuivre l'effort dans le même sens. La pédagogie ne doit toutefois pas souffrir de cette solution, a priori séduisante à plusieurs points de vue. Mise à disposition de ressources ne rime pas avec formation à distance, en tout cas pas dans le contexte des retours d'expérience présentés ici.

\section{OPTION TRANSVERSALE «SETRE »}

La première session de la formation optionnelle transversale dite «systèmes embarqués temps-réels » s'est déroulée de février à mars 2006. L'OT SETRE se déroule sur 8 semaines, à raison de $96 \mathrm{~h}$ de face-à-face pédagogique. Les étudiants n'ont pas déclarés la réalité du nombre d'heure qu'ils ont consacrés au projet, mais la qualité des restitutions laisse penser qu'il s'agit d'un investissement important. Une équipe pluridisciplinaire de 10 enseignants encadre la formation avec une forte exigence d'implication de la part des étudiants et de qualité au final. Par nature optionnelle, l'OT SETRE doit son bon déroulement à l'adhésion des étudiants, qui y trouvent donc une valeur ajoutée. Cet engouement se vérifie dans les évaluations au détail près des aspects ressources.

\subsection{Résumé du contenu pédagogique}

L'idée originelle est d'inciter les étudiants à partager leurs compétences, s'approprier celles de leurs co-équipiers, pour construire un produit applicatif répondant à un cahier des charges, et en suivant une méthodologie acceptée dans l'industrie. Au fil des semaines (tableau 1) se déroulent des cours que les étudiants capitalisent par une application directe à leur projet. La figure 1 illustre que partant des aspects matériels, la progression en 5 piliers, va vers la méthodologie. En parallèle, du point de vue pratique, les étudiants s'approprient les équipements support de leur projet, l'environnement de développement, jusqu'à quelques méthodes qu'ils mettent en œuvre immédiatement avec différentes productions de préparation et suivi de projet.

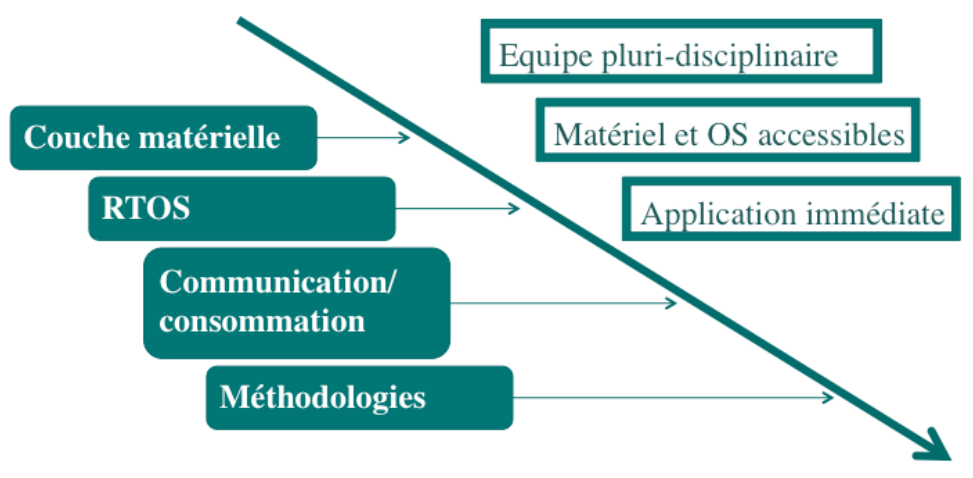

Fig 1 : Progression de la formation

La figure 2 résume le contenu pédagogique de la formation. Le tableau 1 donne les temps forts de la progression de la formation. L'idée n'est pas d'entrer dans les détails techniques de l'état de l'art. 


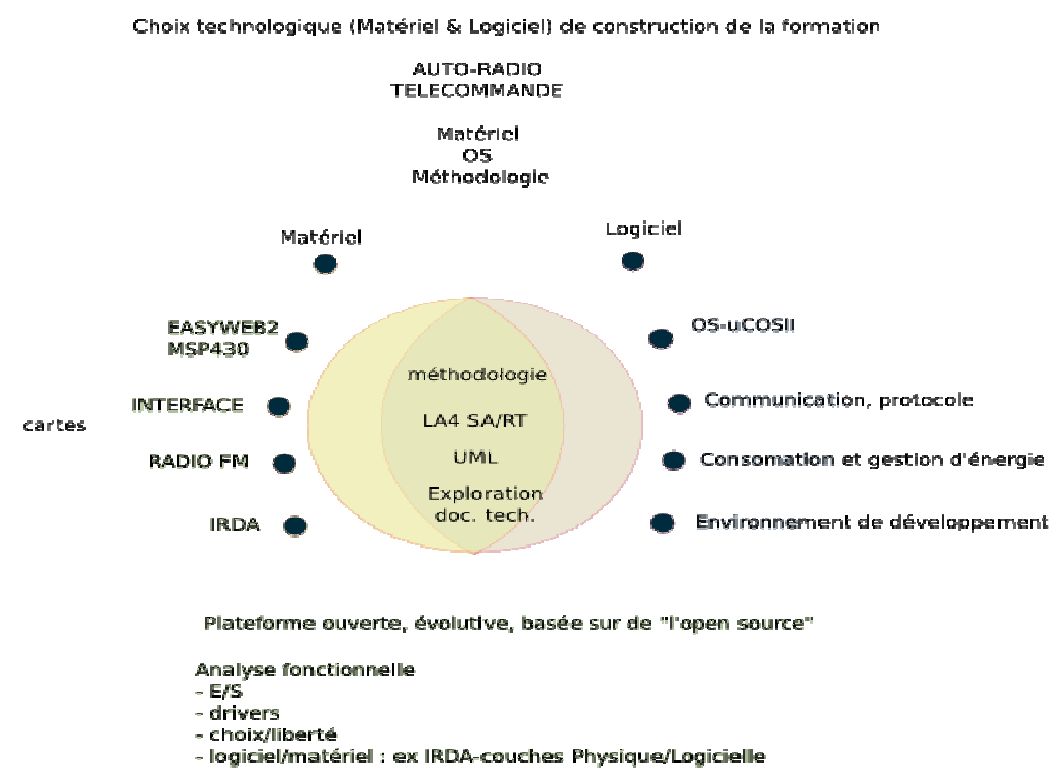

Fig 2 : Contenu pédagogique de la formation

Il s'agit de couvrir, avec des choix arbitraires mais réalistes, différents points d'un système embarqué. Les étudiants sont guidés vers le développement d'un applicatif logiciel qui les utilise. Pour développer cet applicatifs, les étudiants doivent se référer au matériel, voire le configurer, pratiquer des mesures (bus logiques, consommation) et des calibrations (canaux FM par exemple) et tailler sur mesure le système d'exploitation car la mémoire disponible est contrainte.

Tableau 1 : Jalons principaux de la formation OT SETRE

\begin{tabular}{|l|c|c|}
\hline \multicolumn{2}{|c|}{ Apports théoriques } & Projet fil rouge \\
\hline S1 & $\begin{array}{c}\text { Introduction, Architectures maté- } \\
\text { rielles, rappel système d'exploitation (OS) }\end{array}$ & $\begin{array}{c}\text { Découverte du cahier des charges et de } \\
\text { l'environnement de développement }\end{array}$ \\
S2 & $\begin{array}{c}\text { Méthodologie de conception (apports } \\
\text { différenciés) }\end{array}$ & $\begin{array}{c}\text { Travaux Pratiques d'apprentissage des } \\
\text { outils dont le simulateur lié au projet, mise } \\
\text { en ceuvre de la méthodologie, rendu de ca- } \\
\text { hiers de préparation }\end{array}$ \\
\hline S3 & $\begin{array}{c}\text { Approfondissements OS et introduc- } \\
\text { tion du produit uC-OS2 }\end{array}$ & $\begin{array}{c}\text { Analyse et conception autour de uC- } \\
\text { OS2, rendu de cahiers de conception }\end{array}$ \\
\hline S4 & $\begin{array}{c}\text { Communication et pilotes de périphé- } \\
\text { riques }\end{array}$ & $\begin{array}{c}\text { Mise en oeuvre de la communication } \\
\text { par IrDA }\end{array}$ \\
\hline S5 & Communication (suite), validation & Aspects protocole simplifié IrDA \\
\hline S6 & $\begin{array}{c}\text { Intervention industrielle, Optimisation } \\
\text { vis-à-vis de la consommation énergétique }\end{array}$ & Intégration fonctionnelle et tests \\
\hline S7 & $\begin{array}{c}\text { Méthodologie de conception logi- } \\
\text { cielle: approche objet et MDA }\end{array}$ & Tests et Validation \\
\hline S8 & $\begin{array}{c}\text { Intervention industrielle et restitution } \\
\text { orale des projets. }\end{array}$ & Démonstration et rapport final \\
\hline
\end{tabular}

\subsection{Ressources matérielles}

La première session de l'OT SETRE avait pour but d'évaluer la pertinence du contenu pédagogique, et en conséquence s'est appuyée sur des ressources économiques. L'open-source a été de 
mise pour l'environnement logiciel de développement applicatif (mspgcc, mspdebug, Free-RTOS puis $\mu \mathrm{C}$-OSII). Ceci a orienté le choix de la version du microcontrôleur par exemple, donc l'ambition du projet de fil. La carte très artisanale de cette première session n'a survécu qu'un an, tout en ayant permis aux étudiants d'atteindre un objectif ambitieux (figure 3) : réaliser un récepteur FM, une IHM lisible, pilotable à distance et enregistrant des statistiques d'usage. Ce projet fil rouge a été conservé, tout en laissant un degré de liberté aux étudiants sur les aspects méthodologies (ouverture aux méthodes agiles par exemple).

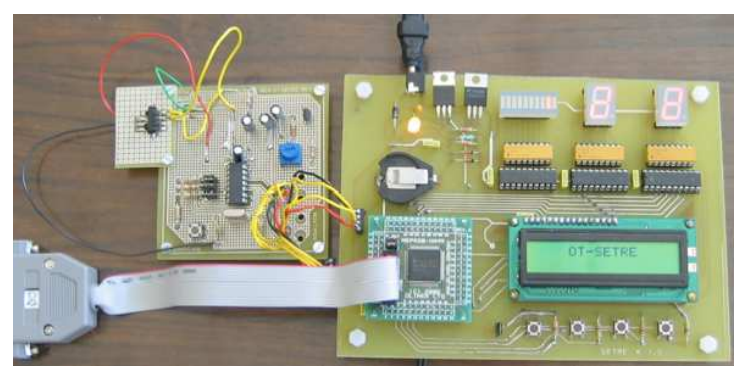

Fig 3 : Carte lego économique des débuts

Le dispositif Bonus Qualité Formation de l'INSA de Lyon a financé la construction de cartes lego pour la poursuite de l'OT. La figure 4 montre l'aspect un peu moins artisanal, permettant aux étudiants de bricoler si besoin. Le retour des étudiants est sans appel : si les cartes remplissent leurs objectifs, leur qualité et leur fiabilité n'est pas au rendez-vous. Par ailleurs, chaque étudiant aurait souhaité disposer d'un lot suffisant de cartes (communication entre cartes). Ceci est de moins en moins possible puisque le stock diminue d'année en année. C'est la motivation d'un changement d'approche dans la mise à disposition des ressources matérielles et logicielles.

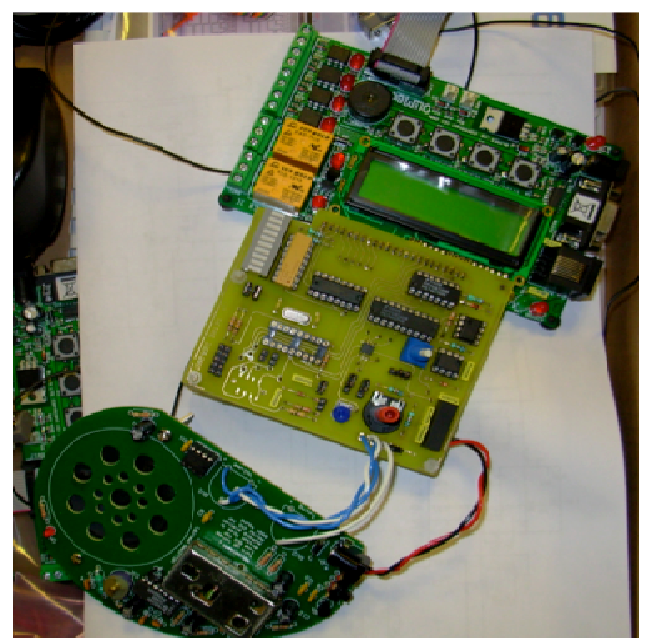

Fig 4 : Carte lego économique ayant survécu 5 ans

\section{RESSOURCES ACCESSIBLES À DISTANCE}

Pour pallier le souci de fiabilité, il a été fait le choix de cartes standard de développement, tierce partie (figure 5). Des cartes filles permettent d'élaborer divers objectifs applicatifs (figure 6) : la pédagogie n'est pas remise en cause.

Pour augmenter la disponibilité des ressources matérielles, un projet actuel vise à les rendre accessibles à distance via internet. Le synoptique du projet est schématisé sur la figure 7.

Cette idée n'est pas nouvelle [1]. De nombreux appareils de mesure sont maintenant accessibles à distance, appuyés par des pédagogies différentes [2-4]. Un portail d'accès gère les connec- 
tions et les droits. L'AIP-PRIMECA, sur le même campus, a l'expérience de télé-TPs et chapeautera nos besoins sous un portail unique (dépôt de projet SRESRI, 2013). L'idée ici n'est pas de rappeler les bénéfices et limitations d'une approche d'enseignement à distance mais d'indiquer nos objectifs à l'appui de cette ressources supplémnetaires.

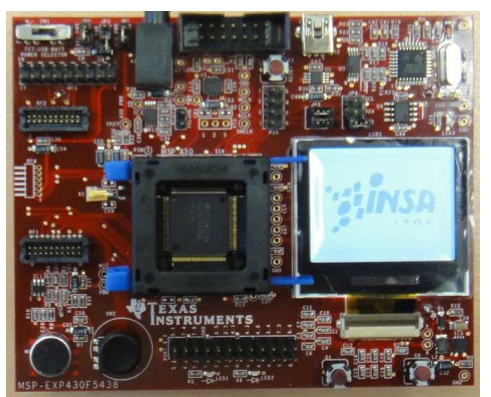

Fig 5 : Carte de développement standard
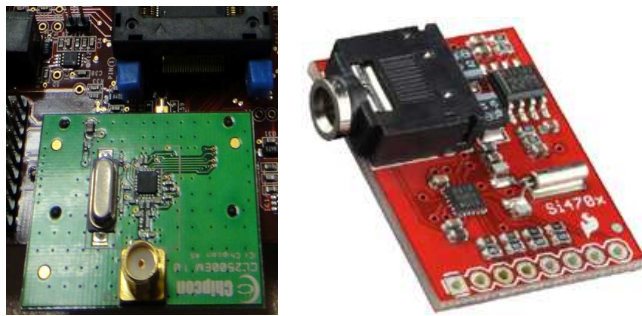

Fig 6 : Cartes filles de communication

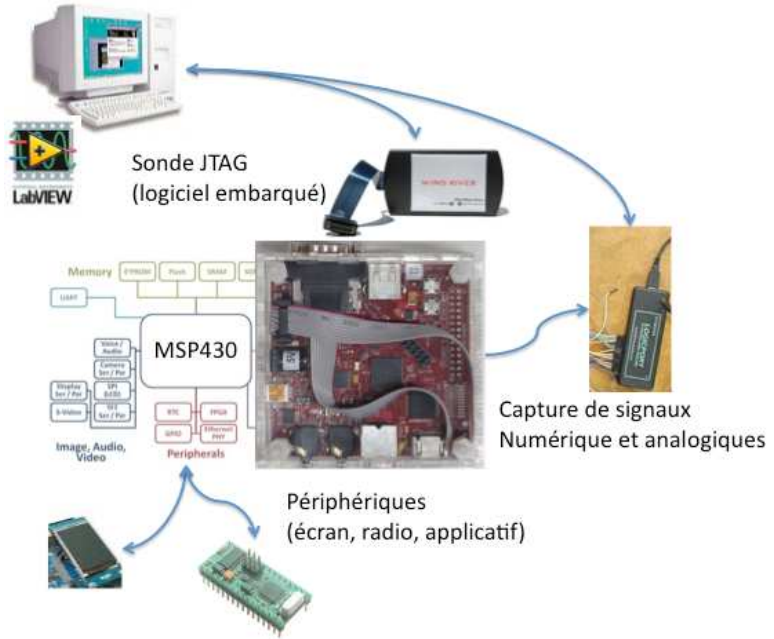

Fig 7 : Synoptique d'accès à distance des ressources

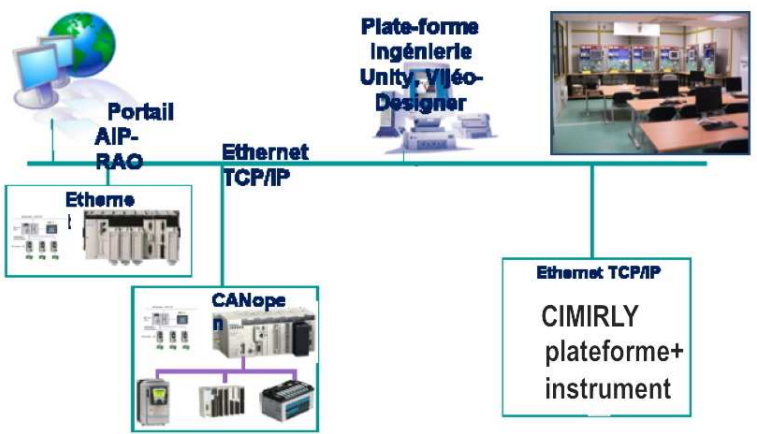

Fig 8 : Organisation de la gestion de l'accès à distance à l'AIP Primeca 
Nous concevons la ressource accessible à distance comme un complément offert aux étudiants pour prolonger leur travail en dehors des murs. Afin de limiter les développements informatiques, nous avons décidé d'un accès à un bureau éloigné, situé sur une machine frontale. Celle-ci sera identique aux machines sur lesquelles les étudiants utilisent la suite logicielle de développement microcontrolleur et les instruments de mesure (oscilloscope, analyseur logique).

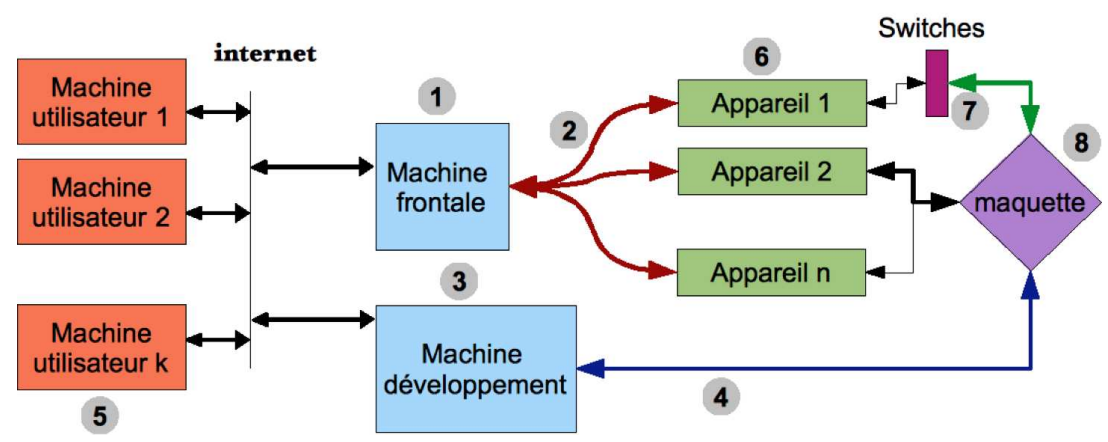

Fig 9 : Synoptique fonctionnel

Ce principe permet d'aller au-delà. Notamment des instruments non duplicables pour des raisons de coût, trouvent une utilisation pratique dans ce cadre. Par exemple la mise en œuvre de capteurs de mesure dédiés, sur une carte modifiée, va permettre l'accès corréler à l'applicatif logiciel et à la consommation énergétique du système. Ceci n'est pas praticable car il est à craindre une perte de fiabilité à modifier toutes les cartes matérielles en ce sens. D'ailleurs l'exercice, non compris dans le contenu initial de la formation, doit être vu comme un sujet supplémentaire. Dans ce cas, une pédagogie adaptée doit être offerte (enseignement à distance) contrairement à l'usage de «prolongation ».

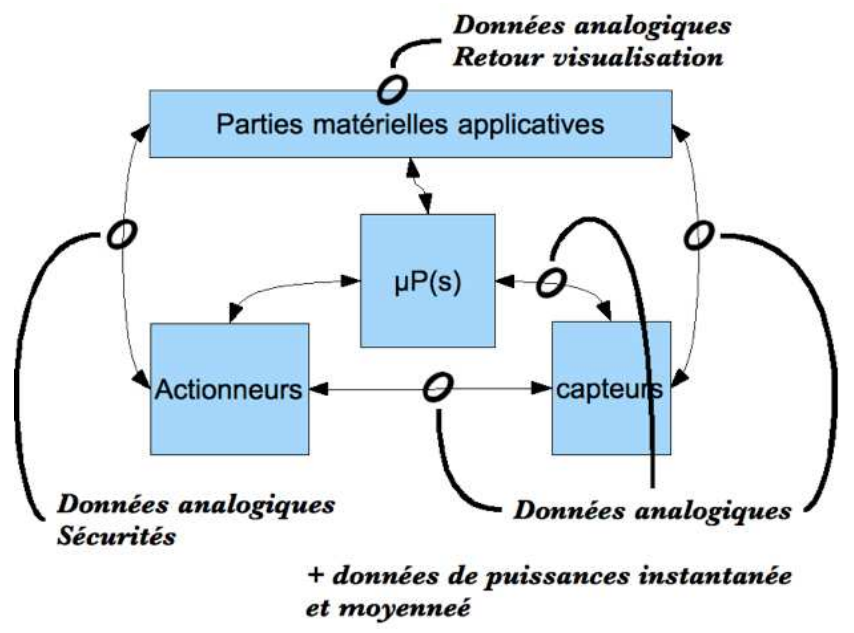

Fig 10 : Sujet supplémentaire

La Région Rhône-Alpes a contribué, en 2012, à l'achat de l'équipement (serveurs, cartes, instrumentation). La suite de développement IAR Workbench a été sélectionnée vis-à-vis du microcontrôleur (MSP430). Le projet a atteint la phase de mise en place pour un accès à distance. Une séance de travaux pratiques est néanmoins en cours de préparation. Dans l'annexe 1, le lecteur trouvéra le sujet ainsi que la correction de cette séance. 


\section{SECOND PROJET DE TÉLÉ-TP}

Poursuivant l'idée de la mise à disposition plus large de moyens matériels, une deuxième plate-forme a été construit en vue d'illustrer la relation entre modulation, transmission RF et consommation énergétique [5]. Cette plate-forme a été financée par l'INSA à travers le dispositif BQF.

Pour les élèves ayant étudié les briques de base de la radiocommunication, le banc leur propose de se familiariser par la pratique avec certains paramètres quantifiant la qualité de la communication tels que l'EVM ou le taux d'erreurs binaire (BER). Pour ce faire, la plate-forme contient un émetteur et un récepteur réalisés à l'aide des cartes d'évaluation du circuit intégré Maxim 2830.

Le transceiver MAXIM 2830 contient un émetteur et un récepteur, sur la bande $2,4-2,5 \mathrm{GHz}$ et offre une bande passante maximale de $20 \mathrm{MHz}$ pour les signaux véhiculés, ceci offrant une flexibilité pour la modulation des signaux. Un générateur vectoriel de signaux ESG E4432B permet la synthèse de modulation. Le chemin d'émission peut être perturbé à loisir pour fixer un niveau d'atténuation et de bruit. Les signaux transposés après réception, sont traités par un numériseur L4532A.

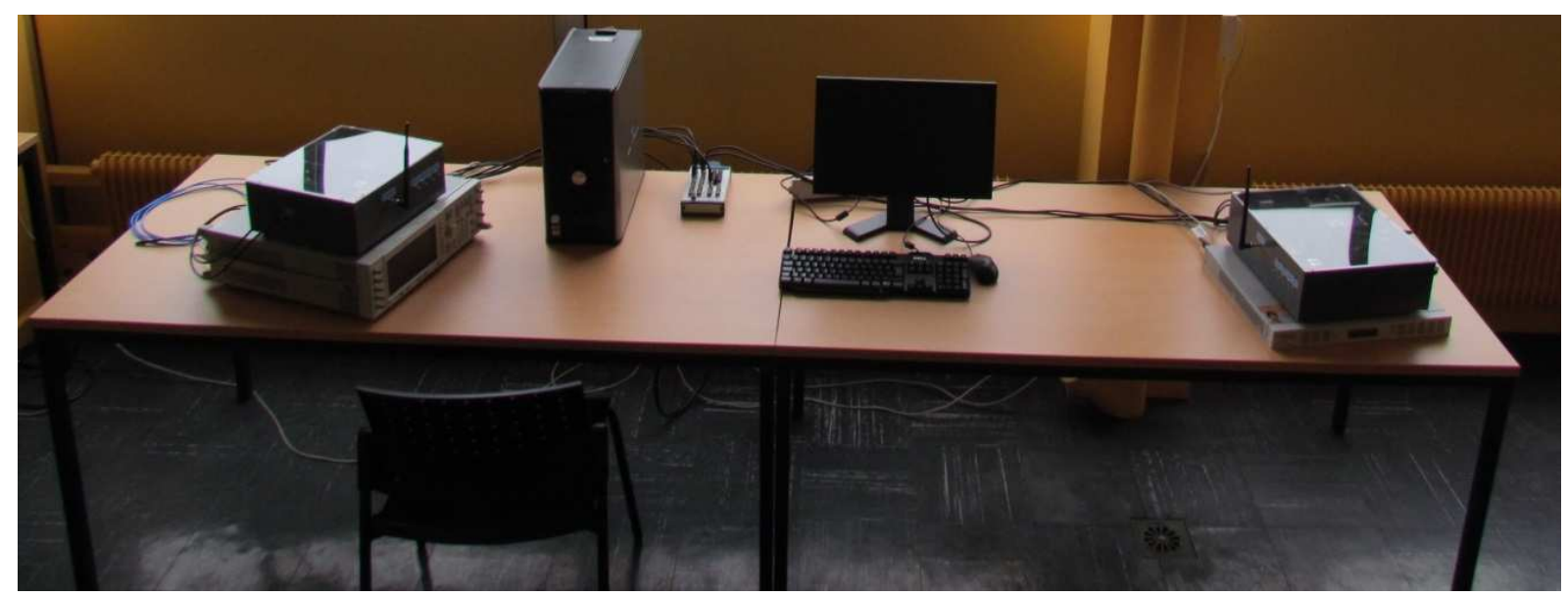

Fig 11 : Banc de test radiocommunication

Des cartes d'acquisition permettent de saisir des indicateurs de consommation, indépendamment du fonctionnement du système. Le rapprochement des deux types de résultat permet alors de corréler modulation, paramètres d'émission, débits effectifs, qualité et consommation.

Une machine frontale gère le banc et son accès à distance rend le banc utilisable hors les murs. Il est prévu une utilisation du banc en prolongement de travaux pratiques que les étudiants effectueront sur un banc similaire. Des exercices supplémentaires pourront alors être proposés à titre complémentaire.

Une séance de travaux pratiques proposée (voir annexe 2) permet d'appréhender la problématique de la génération des signaux numérique, leur transmission par voie hertzienne et leur réception. Plusieurs signaux sont analysés, à partir des modulations simples jusqu'à des signaux correspondant à des normes de communication évoluées, comme la 802.11a.

La figure 12 montre les différents paramètres d'un signal BPSK reçu et traité par la plateforme. Ainsi les élèves ont la possibilité de comparer avec la théorie l'allure fréquentielle, temporelle et la constellation du signal reçu. Ils sont également confrontés à la problématique de l'indétermination de la phase du signal reçu et les paramètres quantifiant la qualité de la communication : l'EVM ainsi que le TEB sont introduits. 
Sur la figure 13 il est présenté le cas d'une démodulation d'un signal de la norme 802.11a. Les différentes parties de la trame ainsi que le type de modulation pour les données ainsi que pour les pilotes sont facilement repérables.

La plate-forme dispose également d'un système de mesure de la distance ; en utilisant des tensions continues proportionnelles à la puissance du signal émis ainsi qu'à la puissance du signal reçu (RSSI), les élèves sont amenés à effectuer un bilan de liaison et de confronter les résultats obtenus à la formule de Friis.
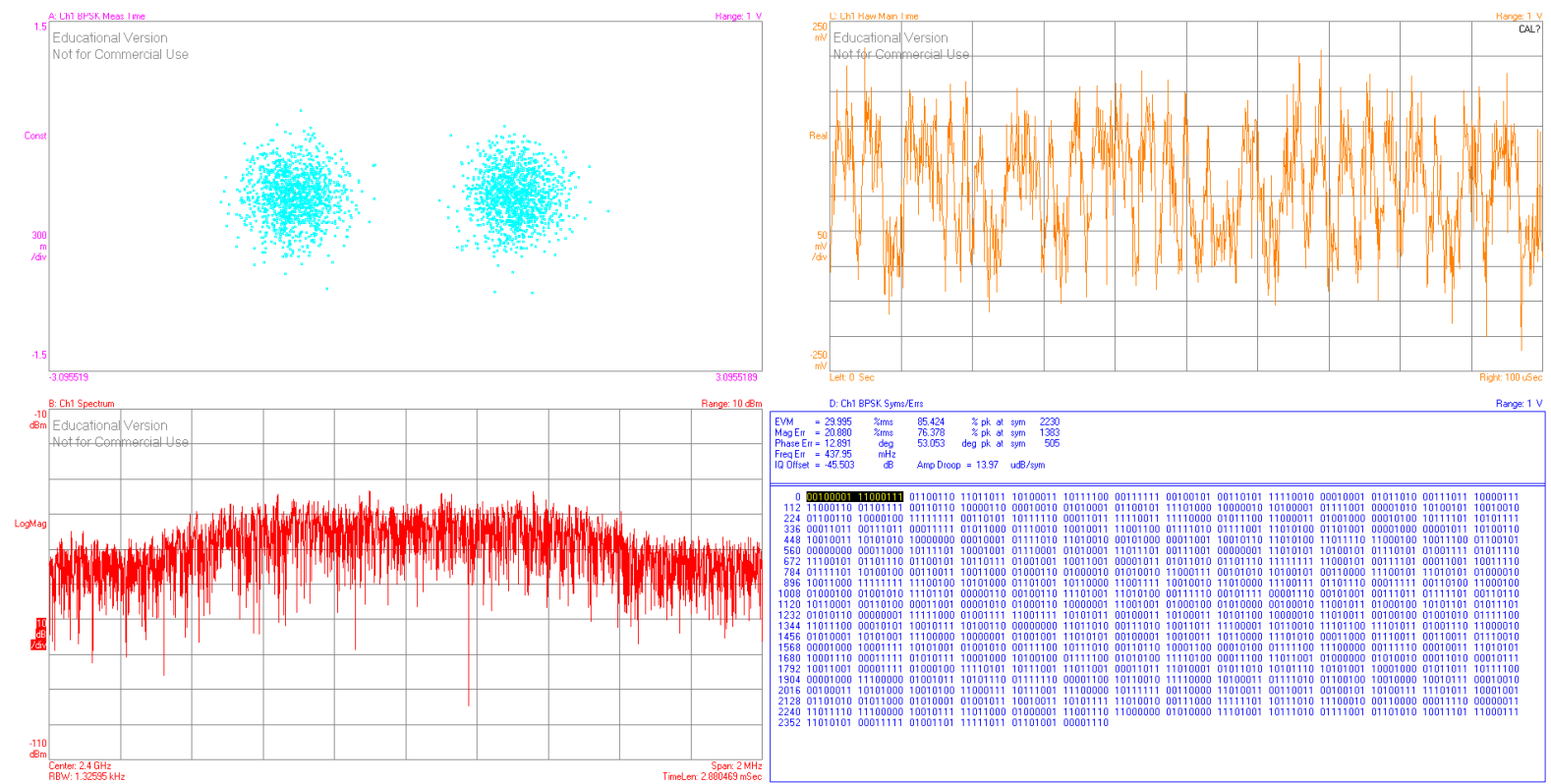

Fig 12 Réception d'un signal BPSK
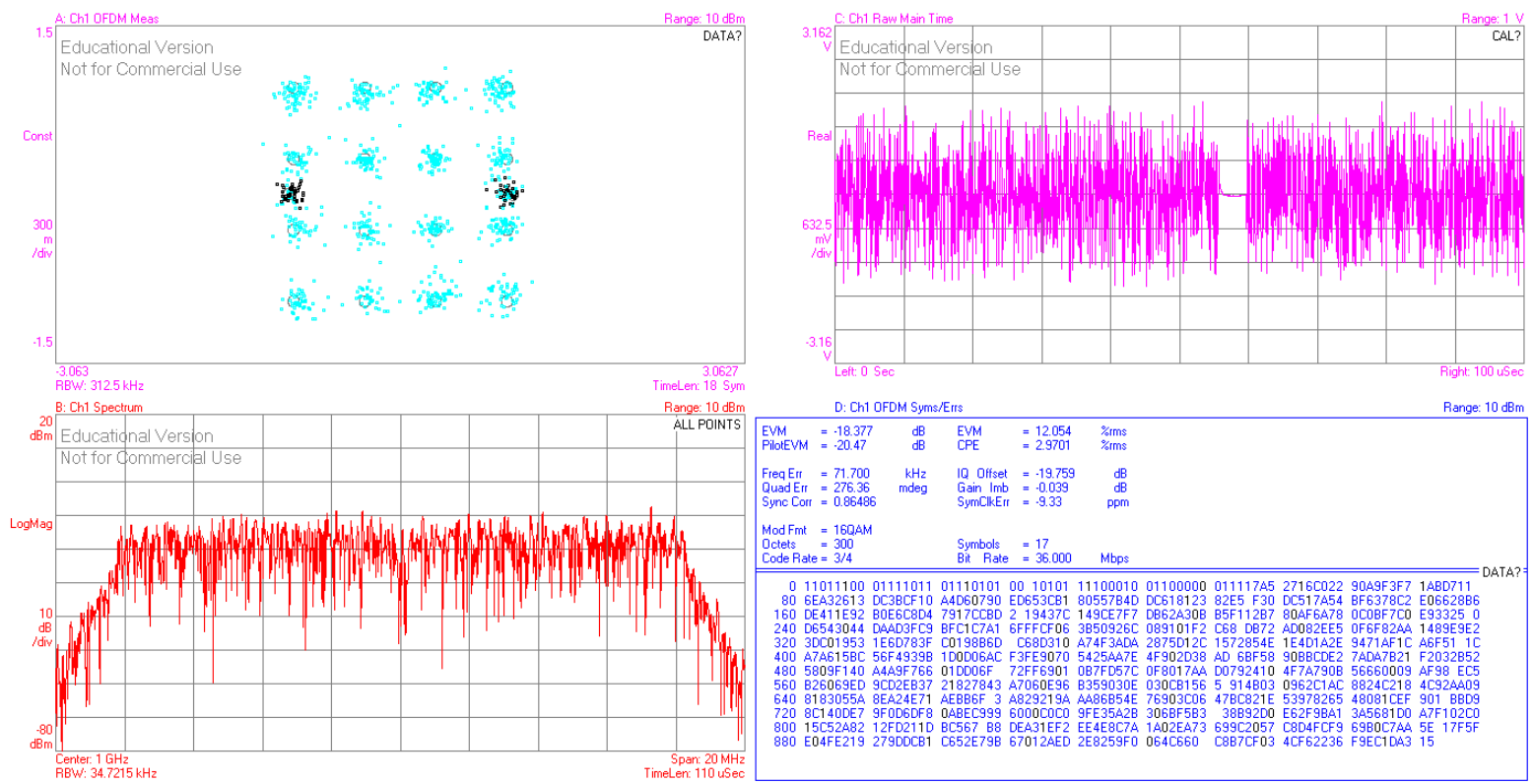

Fig 13 Réception d'un signal 802.11a

L'idée n'est pas encore celle d'un enseignement à distance. La première difficulté est la sécurisation de la plate-forme. Ceci est également vrai pour le projet de la figure 7 . Trop de scenarii sont 
à prévoir avant de penser pouvoir laisser l'accès libre à des telles ressources. Le fait que les étudiants aient manipulés, sous encadrement, ces bancs, réduit fortement le risque.

Dans des configurations réduites, il est envisagé l'utilisation du banc en libre accès à distance, à des fins d'initiation à des concepts simples, comme l'observation d'une constellation classique par exemple (ou la programmation d'un timer de microcontrôleur, autre exemple).

\section{CONCLUSION}

Cet article a présenté la vie d'une formation où la qualité des ressources matérielles est essentielle. Faute de moyens suffisants, l'accès à distance à ces ressources résout le problème de la disponibilité. Sans impacter la pédagogie, ceci permet de proposer des exercices complémentaires ou bien l'accès à des moyens matériels particuliers en nombre insuffisant pour les considérer dans le cadre d'un face-à-face. Il s'agit donc de faire mieux pour le service des étudiants sans remettre en cause une pédagogie qui porte ses fruits par ailleurs.

\section{REMERCIEMENTS}

L'équipe enseignante remercie Régis Aubry, collègue parti récemment en retraite pour son investissement dans l'OT SETRE. L'équipe remercie la Région Rhône-Alpes pour son soutien aux expériences pédagogiques (SRESR 2011, SRERI 2012), en complément du dispositif Bonus Qualité Formation de l'INSA Lyon.

\section{BIBLIOGRAPHIE}

1 - Florin HUTU, florin-doru.hutu@insa-lyon.fr, enseignant au département Génie Electrique (http://ge-insa-lyon.fr), chercheur au laboratoire CITI à l'INSA de Lyon ( http://www.citi$\underline{\text { lab.fr/) }}$

2 - Bruno ALLARD, bruno.allard@insa-lyon.fr, enseignant au département Génie Electrique (http://ge-insa-lyon.fr), chercheur au laboratoire Ampère à l'INSA de Lyon ( http://www.ampere-lab.fr/)

3 - Fabrice JUMEL, jumel@cpe.fr, enseignant à Chimie Physique Electronique (CPE Lyon, http://www.cpe.fr/-Les-laboratoires-electronique-.html), chercheur au laboratoire CITI à l'INSA de Lyon ( http://www.citi-lab.fr/).

4 - Mathieu MARANZANA, mathieu.maranzana@insa-lyon.fr, enseignant chercheur au département Informatique ( http://if.insa-lyon.fr), responsable des études

5 - Kevin MARQUET, kevin.marquet@insa-lyon.fr, enseignant au département Informatique (http://if-insa-lyon.fr ), chercheur au laboratoire CITI à l'INSA de Lyon ( http://www.citi$\underline{\mathrm{lab} . \mathrm{fr} /}$ ).

6 - Lionel MOREL lionel.morel@insa-lyon.fr, enseignant au département Informatique (http://ifinsa-lyon.fr), chercheur au laboratoire CITI à l'INSA de Lyon ( http://www.citi-lab.fr/).

7 - Luong-Viet PHUNG enseignant au département Génie Electrique (http://ge-insa-lyon.fr), chercheur au laboratoire Ampère à l'INSA de Lyon ( http://www.ampere-lab.fr/)

8 - Tanguy RISSET enseignant au département Télécommunications et usages (http://telecom.insalyon.fr), chercheur au laboratoire CITI à l'INSA de Lyon ( http://www.citi-lab.fr/).

9 - Dominique TOURNIER enseignant au département Génie Electrique (http://ge-insa-lyon.fr), chercheur au laboratoire Ampère à l'INSA de Lyon ( http://www.ampere-lab.fr/)

10 - Guillaume SALAGNAC enseignant au département Informatique (http://if-insa-lyon.fr), chercheur au laboratoire CITI à l'INSA de Lyon ( http://www.citi-lab.fr/).

11 - Jacques VERDIER enseignant au département Génie Electrique (http://ge-insa-lyon.fr), chercheur au laboratoire Ampère à l'INSA de Lyon ( http://www.ampere-lab.fr/) 


\section{RÉFÉRENCES}

[1] Jacques Fayolle, "De la recherche en traitement d'images à la recherche d'informations en passant par l'enseignement à distance et le travail collaboratif', Habilitation à Diriger des Recherches, Université Jean Monnet, 2007.

[2] B. Bayard, B. Sauviac, J. Fayolle, B. Allard, G. Noyel, "Projet WebAnalyzer Internet et l'instrumentation à distance", Technologies de l'information et de la communication dans l'Enseignement Supérieur et l'Entreprise, TICE, INSA Lyon 13-15 Novembre 2002 , pp. 415416

[3] GIP CNFM, "Centre de ressources de test", http://web.cnfm.fr/PCM/CRTC/

[4] P. Kadionik, T. Zimmer, Y. Danto, "RETWINE : A new distributed Environment for Microelectronics Intrumentation Learning and Measurement", IEEE Instrumentation and Measurement Technology Conference, Italy (1999)

[5] F. Hutu, J.Verdier, G. Villemaud, B. Allard, "Mise en place d'une plate-forme de radiocommunications numériques", Journées pédagogiques du CNFM, Saint-Malo, 28-30 novembre 2012. 